\title{
Canonical approach to the closed string non-commutativity
}

\author{
Lj. Davidovića ${ }^{\text {, B. Nikolicé }}$, B. Sazdovićc \\ Institute of Physics, University of Belgrade, P.O.Box 57, 11001 Belgrade, Serbia
}

Received: 27 November 2013 / Accepted: 20 December 2013 / Published online: 28 January 2014

(C) The Author(s) 2014. This article is published with open access at Springerlink.com

\begin{abstract}
We consider the closed string moving in a weakly curved background and its totally T-dualized background. Using T-duality transformation laws, we find the structure of the Poisson brackets in the T-dual space corresponding to the fundamental Poisson brackets in the original theory. From this structure we see that the commutative original theory is equivalent to the non-commutative T-dual theory, whose Poisson brackets are proportional to the background fluxes times winding and momentum numbers. The noncommutative theory of the present article is more nongeometrical than T-folds and in the case of three space-time dimensions corresponds to the nongeometric space-time with $R$-flux.
\end{abstract}

\section{Introduction}

It is well known that the open string endpoints, attached to a $D p$-brane, are non-commutative [1-12]. The noncommutativity is implied by the fact that for the solution of the boundary conditions the initial coordinate is given as a linear combination of the effective coordinate and the effective momentum, which have a nonzero Poisson bracket (PB). In the constant background case, the coefficient in front of the momenta is proportional to the Kalb-Ramond field $B_{\mu \nu}$, whose presence is crucial in gaining the non-commutativity.

The closed string does not have endpoints and in the flat space the boundary conditions are satisfied automatically. But, to understand the closed string non-commutativity, we are going to use a explanation similar to the open string case. We will express the closed string coordinates in terms of the coordinates and momenta of some other space. The relation between different spaces will be established using the T-duality transformations.

\footnotetext{
a e-mail: ljubica@ipb.ac.rs

b e-mail: bnikolic@ipb.ac.rs

c e-mail: sazdovic@ipb.ac.rs
}

The T-dualization along isometry directions, and the construction of T-dual theory was first realized through a Buscher procedure $[13,14]$. The procedure is in fact a localization of the translation invariance symmetry, in which beside the covariantization of derivatives one adds the Lagrangian multiplier term to the action, which ensures the physical equivalence of the initial and the T-dual theory.

In flat space, T-duality relates $\sigma$-derivatives of the coordinates of the original theory with the momenta of its T-dual theory, and vice versa. As the momenta of the original theory are taken to be commutative, it follows that the coordinates commute as well. So, in flat space there is no noncommutativity of the closed string T-dual coordinates. This is in agreement with the fact that T-duality is a canonical transformation in the flat space, and with the fact that PB's are invariant under such transformations.

The closed string non-commutativity was first observed in the papers [15], and investigated further in [16-20], where it was found that the commutators of the coordinates are proportional to the flux and the winding number.

Let us briefly describe the result of Ref. [16], following its notation. After $T_{1}$-dualization along the $X^{1}$ coordinate, one obtains the twisted torus with coordinates $Y^{a}(a=1,2,3)$ and $f$-flux. After additional $T_{2}$-dualization along $X^{2}=Y^{2}$ one obtains the nongeometric background with coordinates $Z^{a}$ and $Q$-flux. Using the standard Buscher prescription one cannot perform $T_{3}$-dualization along the coordinate $X^{3}=$ $Y^{3}=Z^{3}$ because the Kalb-Ramond field $B_{a b}$ depends on $Z^{3}$. But it is argued in Refs. $[16,21,22]$ that $T_{3}$-dualization leads to a nongeometric background with R-flux configuration and $W^{a}$ coordinates presented in the T-duality chain,

$H_{a b c}, X^{a} \stackrel{T_{1}}{\rightarrow} f_{b c}^{a}, Y^{a} \stackrel{T_{2}}{\rightarrow} Q_{c}^{a b}, Z^{a} \stackrel{T_{3}}{\rightarrow} R^{a b c}, W^{a}$

In the paper [16], the non-commutativity of the nongeometric background ( $Z^{a}$ with $Q$-flux) has been obtained using its $T_{2}$-duality connection $Z^{a}=Z^{a}\left(Y^{a}\right)$ with the geometric background (twisted torus with $Y^{a}$ and $f$-flux). 
In our paper [23], we performed a generalized Buscher T-dualization procedure along all the coordinate directions. It corresponds to the $T=T_{1} \circ T_{2} \circ \cdots \circ T_{D}$-duality relation $y_{\mu}=y_{\mu}\left(x^{\mu}\right)$, connecting the beginning and the end of the T-duality chain:

$$
\begin{aligned}
& H_{\mu \nu \rho}, x^{\mu} \stackrel{T_{1}}{\rightarrow}\left(f_{1}\right)_{\mu \nu \rho}, x_{1}^{\mu} \stackrel{T_{2}}{\rightarrow}\left(f_{2}\right)_{\mu \nu \rho}, x_{2}^{\mu} \stackrel{T_{3}}{\rightarrow} \cdots \\
& \quad \stackrel{T_{D}}{\longrightarrow}\left(f_{D}\right)_{\mu \nu \rho}, x_{D}^{\mu}=y_{\mu},
\end{aligned}
$$

where $\left(f_{i}\right)_{\mu \nu \rho}$ and $x_{i}^{\mu},(i=1,2, \ldots, D)$ are fluxes and the coordinates of the corresponding configuration. In $D$ dimensional space-time it is possible to perform T-duality along any subset of coordinates. For simplicity, in the present article we will T-dualize all the directions. The general case will be published separately.

We considered the bosonic string moving in a background with constant metric $G_{\mu \nu}=$ const and the linear KalbRamond field $B_{\mu \nu}=b_{\mu \nu}+\frac{1}{3} B_{\mu \nu \rho} x^{\rho}$, where the field strength of the Kalb-Ramond field $B_{\mu \nu \rho}$ is infinitesimally small (for more details see the introductory part of Sect. 2). The T-dual theory obtained is of the same form as the initial theory, so that the T-dual string moves in the T-dual background, but in the doubled space given by the coordinates $y_{\mu}, \tilde{y}_{\mu}$. The dual coordinates satisfy the following conditions: $\dot{y}_{\mu}=\tilde{y}_{\mu}^{\prime}, y_{\mu}^{\prime}=\dot{\tilde{y}}_{\mu}$. The improvement, in comparison to the standard Buscher procedure, is the covariantization of the coordinates $x^{\mu}$. In fact, because $x^{\mu}$ is gauge dependent, it is replaced by the gauge invariant expression $\Delta x_{i n v}^{\mu}=\int \mathrm{d} \xi^{\alpha} D_{\alpha} x^{\mu}$. As pointed out in [21,22], the T-dual background of the present paper is of the 'new class that is even more nongeometrical than $T$-folds'. Unlike the T-folds, this background is not a standard manifold even locally. In our formulation, this stems from the fact that the argument of the background fields $\Delta x_{i n v}^{\mu}$ is the line integral. Some authors argued that such a spaces (for $D=3$ known as R-flux background) involve nonassociative geometries [24].

In the canonical formalism, the T-dual variables can be expressed in terms of the original ones in the simple form $y_{\mu}^{\prime} \cong \frac{1}{\kappa} \pi_{\mu}-\beta_{\mu}^{0}[x]$ and ${ }^{\star} \pi^{\mu} \cong \kappa x^{\prime \mu}+\kappa^{2} \theta_{0}^{\mu \nu} \beta_{\nu}^{0}[x]$. The infinitesimal expression $\beta_{\mu}^{0}$ is an improvement in comparison to the flat background case. Because the coordinates and momenta of the original theory do not commute, $\beta_{\mu}^{0}$ is the source of the closed string non-commutativity.

We will follow the main idea of Ref. [16], using the Tduality transformation laws between the T-dual backgrounds in order to study the non-commutativity of the coordinates. In the paper [16], the $T_{2}$-duality connects coordinates $Z^{a}=$ $Z^{a}\left(Y^{a}\right)$ of the nongeometric background ( $Z^{a}$ with $Q$-flux) and the geometric background (twisted torus with $Y^{a}$ and $f$-flux). We performed the T-dualization procedure along all the coordinates, and we obtained the T-duality transformation $y_{\mu}=y_{\mu}\left(x^{\mu}\right)$ of the locally nongeometric background (the end of the chain (1.2) with $y_{\mu}$ and $f_{D}$-flux) and the geometric background (torus with $H$-flux in the beginning of the chain (1.2)). In both approaches it was assumed that the geometric backgrounds (described by $Y^{a}$ in [16] and by $X^{a}$ in our paper) have the standard commutation relations. The PB between the $y_{\mu}$ is proportional to the flux $B_{\mu \nu \rho}$ and the winding number $N^{\mu}$ of the initial theory. In addition, we obtain the complete algebra of the T-dual coordinates and momenta in terms of the fluxes.

For $D=3$, the case of the present article corresponds to T-duality, $T=T_{1} \circ T_{2} \circ T_{3}$, which connects the coordinates $W^{a}=W^{a}\left(X^{a}\right)$ of the nongeometric background ( $W^{a}$ with $R$-flux) and the geometric background (torus with $X^{a}$ and $H$-flux). In comparison to Ref. [16], this procedure contains one $T$-dualization more, $T_{3}$-dualization along the coordinate $X^{3}=Y^{3}=Z^{3}$, which cannot be done using the standard Buscher prescription because the Kalb-Ramond field $B_{a b}$ depends on $Z^{3}$. Thus, in terms of Ref. [16], we obtained the non-commutativity of the nongeometric background, with R-flux configuration. This background does not look like the conventional space even locally.

At the end we give three appendices. In the first one we derive in detail the expression for the dual momentum ${ }^{\star} \pi^{\mu}$, while in the second one we present a list of the fluxes used in the paper. The third appendix contains the mathematical details regarding the transition from $\mathrm{PB}\{\Delta X, \Delta Y\}$ to $\mathrm{PB}$ $\{X, Y\}$.

\section{Bosonic string in the weakly curved background and its T-dual picture}

Let us consider the closed string moving in the $D$-dimensional space-time, in the coordinate $x^{\mu}(\tau, \sigma), \mu=0, \ldots, D-1$ dependent background, described by the action

$S[x]=\kappa \int_{\Sigma} \mathrm{d}^{2} \xi \partial_{+} x^{\mu} \Pi_{+\mu \nu}[x] \partial_{-} x^{\nu}$.

We suppose that all the coordinates are compact, with radii $R_{\mu}$. The background is defined by the space-time metric $G_{\mu \nu}$ and the antisymmetric Kalb-Ramond field $B_{\mu \nu}$,

$\Pi_{ \pm \mu \nu}[x]=B_{\mu \nu}[x] \pm \frac{1}{2} G_{\mu \nu}[x]$.

The light-cone coordinates are

$\xi^{ \pm}=\frac{1}{2}(\tau \pm \sigma), \quad \partial_{ \pm}=\partial_{\tau} \pm \partial_{\sigma}$,

and the action is given in the conformal gauge (the worldsheet metric is taken to be $g_{\alpha \beta}=e^{2 F} \eta_{\alpha \beta}$ ).

World-sheet conformal invariance is required as a condition of having a consistent theory on the quantum level [25-28]. This results in the following space-time equations 
for the background fields:

$R_{\mu \nu}-\frac{1}{4} B_{\mu \rho \sigma} B_{\nu}^{\rho \sigma}=0, \quad D_{\rho} B_{\mu \nu}^{\rho}=0$,

in the lowest order in the slope parameter $\alpha^{\prime}$ and for the constant dilaton field $\Phi=$ const. Here

$B_{\mu \nu \rho}=\partial_{\mu} B_{v \rho}+\partial_{\nu} B_{\rho \mu}+\partial_{\rho} B_{\mu \nu}$

is the field strength of the field $B_{\mu \nu}$, and $R_{\mu \nu}$ and $D_{\mu}$ are Ricci tensor and the covariant derivative with respect to the space-time metric.

We will consider a weakly curved background $[11,12,16$, 23,29-31] defined by

$G_{\mu \nu}[x]=$ const,

$B_{\mu \nu}[x]=b_{\mu \nu}+h_{\mu \nu}[x]=b_{\mu \nu}+\frac{1}{3} B_{\mu \nu \rho} x^{\rho}$,

$b_{\mu \nu}, B_{\mu \nu \rho}=$ const.

Here, the constant $B_{\mu \nu \rho}$ is infinitesimally small, which, according to $[15,16,18-20]$, means that we will assume that the $D$-dimensional torus is so large that for any $\mu, v, \rho$

$\frac{B_{\mu \nu \rho}}{R_{\mu} R_{\nu} R_{\rho}} \ll 1$,

where $R_{\mu}(\mu=0,1, \ldots D-1)$ are the radii of the torus. For simplicity we will take $R_{0}=R_{1}=\cdots=R_{D-1}$ and rescale the background fields according to Appendix A of Ref. [16]. The background (2.6) is the solution of Eq. (2.4) in the first order in the $B_{\mu \nu \rho}$ approximation of closed string theory of Eq. (2.1).

\subsection{T-dual bosonic string}

The T-dualization of closed string theory in a weakly curved background was the subject of investigation in [23]. There we presented the T-dualization procedure performed along all the coordinates, in a background which depends on these coordinates. Here we will give a short overview of the most important results.

The T-dual picture of the theory is given by

$$
\begin{aligned}
{ }^{\star} S[y] & =\kappa \int \mathrm{d}^{2} \xi \partial_{+} y_{\mu}{ }^{\star} \Pi_{+}^{\mu \nu}[\Delta V[y]] \partial_{-} y_{\nu} \\
& =\frac{\kappa^{2}}{2} \int \mathrm{d}^{2} \xi \partial_{+} y_{\mu} \Theta_{-}^{\mu v}[\Delta V[y]] \partial_{-} y_{\nu},
\end{aligned}
$$

with

$$
\begin{gathered}
\Theta_{ \pm}^{\mu \nu} \equiv-\frac{2}{\kappa}\left(G_{E}^{-1} \Pi_{ \pm} G^{-1}\right)^{\mu \nu}=\theta^{\mu \nu} \mp \frac{1}{\kappa}\left(G_{E}^{-1}\right)^{\mu \nu}, \\
G_{E \mu \nu} \equiv G_{\mu \nu}-4\left(B G^{-1} B\right)_{\mu \nu} .
\end{gathered}
$$

The dual background fields, defined in analogy with Eq. (2.2) as ${ }^{\star} \Pi_{ \pm}^{\mu \nu}={ }^{\star} B^{\mu \nu} \pm \frac{1}{2}{ }^{\star} G^{\mu \nu}$, have the form

$$
\begin{gathered}
{ }^{\star} G^{\mu v}[\Delta V[y]]=\left(G_{E}^{-1}\right)^{\mu v}[\Delta V[y]], \\
{ }^{\star} B^{\mu \nu}[\Delta V[y]]=\frac{\kappa}{2} \theta^{\mu \nu}[\Delta V[y]] .
\end{gathered}
$$

Using the terminology introduced in the open string case, they are equal to the inverse of the effective metric $G_{\mu \nu}^{E}$ and proportional to the non-commutativity parameter $\theta^{\mu \nu}$. Their argument is given by

$\Delta V^{\mu}[y]=-\kappa \theta_{0}^{\mu v} \Delta y_{v}+\left(g^{-1}\right)^{\mu v} \Delta \tilde{y}_{\nu}$,

where

$$
\begin{aligned}
& \Delta y_{\mu}=\int_{P}\left(\mathrm{~d} \tau \dot{y}_{\mu}+\mathrm{d} \sigma y_{\mu}^{\prime}\right)=y_{\mu}(\xi)-y_{\mu}\left(\xi_{0}\right), \\
& \Delta \tilde{y}_{\mu}=\int_{P}\left(\mathrm{~d} \tau y_{\mu}^{\prime}+\mathrm{d} \sigma \dot{y}_{\mu}\right),
\end{aligned}
$$

and

$g_{\mu \nu}=G_{\mu \nu}-4\left(b G^{-1} b\right)_{\mu \nu}, \quad \theta_{0}^{\mu \nu}=-\frac{2}{\kappa}\left(g^{-1} b G^{-1}\right)^{\mu \nu}$

are constant finite parts of the effective metric and the noncommutativity parameter. The variable $\Delta \tilde{y}_{\mu}$ is path independent on the zeroth order equation of motion. T-dual theory is defined in the doubled space, defined by the two coordinates $y_{\mu}$ and $\tilde{y}_{\mu}$, related by the expressions $\dot{y}_{\mu}=\tilde{y}_{\mu}^{\prime}, y_{\mu}^{\prime}=\dot{\tilde{y}}_{\mu}$.

\subsection{Transformation laws}

The T-duality transformation connecting the variables of the closed string theory in the weakly curved background and its T-dualized string theory is [23]

$\partial_{ \pm} x^{\mu} \cong-\kappa \Theta_{ \pm}^{\mu v}[\Delta V]\left[\partial_{ \pm} y_{v} \pm 2 \beta_{v}^{\mp}[V]\right]$,

with

$\beta_{\mu}^{ \pm}[x]=\frac{1}{2}\left(\beta_{\mu}^{0} \pm \beta_{\mu}^{1}\right)=\mp \frac{1}{2} h_{\mu \nu}[x] \partial_{\mp} x^{\nu}$,

$\beta_{\mu}^{0}[x]=h_{\mu \nu}[x] x^{\prime \nu}, \beta_{\mu}^{1}[x]=-h_{\mu \nu}[x] \dot{x}^{\nu}$.

From Eq. (2.14) we can find the transformation law for $\dot{x}^{\mu}$ and $x^{\prime \mu}$ :

$$
\begin{aligned}
\dot{x}^{\mu \cong \cong} & -\kappa \theta^{\mu v}[\Delta V] \dot{y}_{v}+\left(G_{E}^{-1}\right)^{\mu v}[\Delta V] y_{v}^{\prime}+\left(g^{-1}\right)^{\mu v} \beta_{v}^{0}[V] \\
& +\kappa \theta_{0}^{\mu v} \beta_{v}^{1}[V] \\
x^{\prime \mu} \cong & \left(G_{E}^{-1}\right)^{\mu v}[\Delta V] \dot{y}_{v}-\kappa \theta^{\mu v}[\Delta V] y_{v}^{\prime}-\kappa \theta_{0}^{\mu v} \beta_{v}^{0}[V] \\
& -\left(g^{-1}\right)^{\mu v} \beta_{v}^{1}[V] .
\end{aligned}
$$

Using the expression for the canonical momentum of the original theory,

$\pi_{\mu}=\frac{\delta S}{\delta \dot{x}^{\mu}}=\kappa\left[G_{\mu \nu} \dot{x}^{\nu}-2 B_{\mu \nu}[x] x^{\prime \nu}\right]$, 
and the T-dual canonical momentum,

$$
\begin{aligned}
& { }^{\star} \pi^{\mu}=\frac{\delta^{\star} S}{\delta \dot{y}_{\mu}}=\kappa\left(G_{E}^{-1}\right)^{\mu \nu}[\Delta V[y]] \dot{y}_{v} \\
& -\kappa^{2} \theta^{\mu \nu}[\Delta V[y]] y_{v}^{\prime}-\kappa\left(g^{-1}\right)^{\mu \nu} \beta_{v}^{1}[V[y]],
\end{aligned}
$$

derived in Appendix A, we rewrite the above transformations in canonical form:

$x^{\prime \mu} \cong \frac{1}{\kappa}{ }^{\star} \pi^{\mu}-\kappa \theta_{0}^{\mu \nu} \beta_{v}^{0}[V]$,

$\pi_{\mu} \cong \kappa y_{\mu}^{\prime}+\kappa \beta_{\mu}^{0}[V]$,

with $\beta_{\mu}^{0}[V]$ defined in Eq. (2.15). It is shown in Ref. [23] that the T-dual of the T-dual action is the original one. The corresponding T-dual transformation of the variables law is the inverse of Eq. (2.14),

$\partial_{ \pm} y_{\mu} \cong-2 \Pi_{\mp \mu \nu}[\Delta x] \partial_{ \pm} x^{\nu} \mp 2 \beta_{\mu}^{\mp}[x]$,

and so the transformation laws for $\dot{y}_{\mu}$ and $y_{\mu}^{\prime}$ are equal to

$\dot{y}_{\mu} \cong-2 B_{\mu \nu}[x] \dot{x}^{\nu}+G_{\mu \nu} x^{\prime \nu}+\beta_{\mu}^{1}[x]$,

$y_{\mu}^{\prime} \cong G_{\mu \nu} \dot{x}^{\nu}-2 B_{\mu \nu}[x] x^{\prime \nu}-\beta_{\mu}^{0}[x]$.

Using Eqs. (2.17) and (2.18) we obtain the canonical form of the T-dual transformations,

$y_{\mu}^{\prime} \cong \frac{1}{\kappa} \pi_{\mu}-\beta_{\mu}^{0}[x]$,

${ }^{\star} \pi^{\mu} \cong \kappa x^{\prime \mu}+\kappa^{2} \theta_{0}^{\mu \nu} \beta_{v}^{0}[x]$.

In the zeroth order one has $x^{(0) \mu} \cong V^{\mu}$, and it is easy to see that Eq. (2.22) is the inverse of Eq. (2.17).

Because the T-dual theory is defined in the doubled space, we will need the canonical expression for $\tilde{y}_{\mu}^{\prime}=\dot{y}_{\mu}$. Using Eqs. (2.21a) and (2.17), we obtain

$$
\begin{aligned}
& \tilde{y}_{\mu}^{\prime} \cong-\frac{2}{\kappa}\left(B[\Delta x]+\frac{1}{2} h[x]\right)_{\mu \nu}\left(G^{-1}\right)^{\nu \rho} \pi_{\rho} \\
& +\left(G^{E}[\Delta x]-2 h[x] G^{-1} b\right)_{\mu \nu} x^{\prime \nu} .
\end{aligned}
$$

\section{Non-commutativity relations between canonical variables}

We want to establish the relation between the Poisson structures of the original and T-dual theory. The initial theory is the geometric one, described by the canonical variables $x^{\mu}$ and $\pi_{\mu}$. Thus, we choose the standard form of the PB's in the original space, which are

$$
\begin{gathered}
\left\{x^{\mu}(\sigma), \pi_{v}(\bar{\sigma})\right\}=\delta_{v}^{\mu} \delta(\sigma-\bar{\sigma}), \quad\left\{x^{\mu}(\sigma), x^{\nu}(\bar{\sigma})\right\}=0, \\
\left\{\pi_{\mu}(\sigma), \pi_{\nu}(\bar{\sigma})\right\}=0 .
\end{gathered}
$$

The T-dual theory is the nongeometric one, defined in the doubled space, with two coordinates $y_{\mu}$ and $\tilde{y}_{\mu}$, connected by relations $\dot{y}_{\mu}=\tilde{y}_{\mu}^{\prime}, y_{\mu}^{\prime}=\dot{\tilde{y}}_{\mu}$. Using the T-duality transformation laws, we search for the corresponding Poisson structure in T-dual theory i.e. the expressions for the PB's between the T-dual string coordinates $y_{\mu}(\sigma), \tilde{y}_{\mu}(\sigma)$ and momenta ${ }^{\star} \pi^{\mu}(\sigma)$. This is done considering the brackets between

$\Delta Y_{\mu}\left(\sigma, \sigma_{0}\right)=\int_{\sigma_{0}}^{\sigma} \mathrm{d} \eta Y_{\mu}^{\prime}(\eta)=Y_{\mu}(\sigma)-Y_{\mu}\left(\sigma_{0}\right)$

$Y_{\mu}=y_{\mu}, \tilde{y}_{\mu}$ and calculating the equal time commutators. The fact that T-dual coordinates under T-duality transform to both coordinate and momentum dependent expressions enables non-commutativity. The relation of the form

$\left\{X_{\mu}^{\prime}(\sigma), Y_{\nu}^{\prime}(\bar{\sigma})\right\} \cong K_{\mu \nu}^{\prime}(\sigma) \delta(\sigma-\bar{\sigma})+L_{\mu \nu}(\sigma) \delta^{\prime}(\sigma-\bar{\sigma})$

implies the following relation (derived in Appendix C) between the coordinates

$$
\begin{aligned}
& \left\{X_{\mu}(\tau, \sigma), Y_{\nu}(\tau, \bar{\sigma})\right\} \\
& \quad \cong-\left[K_{\mu \nu}(\sigma)-K_{\mu \nu}(\bar{\sigma})+L_{\mu \nu}(\bar{\sigma})\right] \theta(\sigma-\bar{\sigma}),
\end{aligned}
$$

where $\theta(\sigma)$ is the step function defined in Eq. (8.6).

In flat space the coordinate dependent part of the KalbRamond field is absent, $h_{\mu \nu}=0$, and consequently $\beta_{\mu}^{0}=0$. Thus, from Eqs. (2.22a) and (2.22b) follows $y_{\mu}^{\prime} \cong \frac{1}{\kappa} \pi_{\mu}$ and ${ }^{\star} \pi^{\mu} \cong \kappa x^{\prime \mu}$. Therefore, the PB of the canonical variables of the T-dual theory remain the standard ones, the same as in the original theory. So, the nontrivial infinitesimal expression $\beta_{\mu}^{0}$, which exists only in the coordinate dependent backgrounds, is the source of the closed string non-commutativity.

Using the transformation laws (2.22a) and (2.23), we calculate the PB's $\left\{y_{\mu}^{\prime}, y_{\nu}^{\prime}\right\},\left\{y_{\mu}^{\prime}(\sigma), \tilde{y}_{v}^{\prime}(\bar{\sigma})\right\}$ and $\left\{\tilde{y}_{\mu}^{\prime}(\sigma), \tilde{y}_{v}^{\prime}(\bar{\sigma})\right\}$ and express them in the form of Eq. (3.3) with $\mathrm{K}$ and L equal:

1. $\left\{y_{\mu}^{\prime}, y_{v}^{\prime}\right\}$

$$
K_{\mu \nu}[x]=\frac{3}{\kappa} h_{\mu \nu}[x]=\frac{1}{\kappa} B_{\mu \nu \rho} x^{\rho}, \quad L_{\mu \nu}=0,
$$

2. $\left\{y_{\mu}^{\prime}, \tilde{y}_{\nu}^{\prime}\right\}$

$$
\begin{aligned}
& K_{\mu \nu}[x, \tilde{x}]=\frac{3}{\kappa} h_{\mu \nu}[\tilde{x}]-\frac{6}{\kappa}\left[h[x] G^{-1} b+b G^{-1} h[x]\right]_{\mu \nu}, \\
& L_{\mu \nu}[x]=\frac{1}{\kappa} g_{\mu \nu}-\frac{6}{\kappa}\left[h[x] G^{-1} b+b G^{-1} h[x]\right]_{\mu \nu},
\end{aligned}
$$

with

$\tilde{x}^{\prime \mu}=\frac{1}{\kappa}\left(G^{-1}\right)^{\mu \nu} \pi_{v}+2\left(G^{-1} B\right)_{\nu}^{\mu} x^{\prime \nu}$. 
Using Eqs. (2.6) and (7.2), expressions (3.6) can be rewritten in terms of the fluxes:

$$
\begin{aligned}
& K_{\mu \nu}[x, \tilde{x}]=\frac{1}{\kappa} B_{\mu \nu \rho} \tilde{x}^{\rho}-\frac{3}{2 \kappa} \Gamma_{\rho, \mu \nu}^{E} x^{\rho}, \\
& L_{\mu \nu}[x]=\frac{1}{\kappa} g_{\mu \nu}-\frac{3}{2 \kappa} \Gamma_{\rho, \mu \nu}^{E} x^{\rho},
\end{aligned}
$$

3. $\left\{\tilde{y}_{\mu}^{\prime}, \tilde{y}_{v}^{\prime}\right\}$

$$
\begin{gathered}
K_{\mu \nu}[x]=\frac{3}{\kappa} h_{\mu \nu}[x]+\frac{24}{\kappa}[b h[x] b]_{\mu \nu} \\
+\frac{6}{\kappa}[h[\tilde{x}] b-b h[\tilde{x}]]_{\mu \nu}, \quad L_{\mu \nu}=0 .
\end{gathered}
$$

In terms of fluxes it becomes

$$
\begin{aligned}
& K_{\mu \nu}=-\frac{1}{\kappa}\left[B_{\mu \nu \rho}-6 g_{\mu \alpha} Q_{\rho}^{\alpha \beta} g_{\beta \nu}\right] x^{\rho} \\
& +\left[-\frac{3}{2 \kappa}\left(\Gamma_{\mu, \nu \rho}^{E}-\Gamma_{\nu, \mu \rho}^{E}\right)+\frac{4}{\kappa} B_{\mu \nu \sigma}\left(G^{-1} b\right)_{\rho}^{\sigma}\right] \tilde{x}^{\rho},
\end{aligned}
$$

where $\Gamma_{v, \mu \rho}^{E}$ and $Q_{\mu \nu \rho}$ are defined in Eqs. (7.1) and (7.5).

For the above values of $\mathrm{K}$ and $\mathrm{L}$, the relation (3.4) gives

$$
\begin{aligned}
\left\{y_{\mu}(\sigma), y_{\nu}(\bar{\sigma})\right\} \cong & -\frac{1}{\kappa} B_{\mu \nu \rho}\left[x^{\rho}(\sigma)-x^{\rho}(\bar{\sigma})\right] \theta(\sigma-\bar{\sigma}), \\
\left\{y_{\mu}(\sigma), \tilde{y}_{\nu}(\bar{\sigma})\right\} \cong & -\left\{\frac{1}{\kappa} B_{\mu \nu \rho}\left[\tilde{x}^{\rho}(\sigma)-\tilde{x}^{\rho}(\bar{\sigma})\right]\right. \\
& -\frac{3}{2 \kappa} \Gamma_{\rho, \mu \nu}^{E}\left[x^{\rho}(\sigma)-x^{\rho}(\bar{\sigma})\right] \\
& \left.+\frac{1}{\kappa} g_{\mu \nu}-\frac{3}{2 \kappa} \Gamma_{\rho, \mu \nu}^{E} x^{\rho}(\bar{\sigma})\right\} \theta(\sigma-\bar{\sigma}),
\end{aligned}
$$

$$
\begin{aligned}
\left\{\tilde{y}_{\mu}(\sigma), \tilde{y}_{\nu}(\bar{\sigma})\right\} \cong & -\left\{-\frac{1}{\kappa}\left[B_{\mu \nu \rho}-6 g_{\mu \alpha} Q^{\alpha \beta}{ }_{\rho} g_{\beta \nu}\right]\left[x^{\rho}(\sigma)-x^{\rho}(\bar{\sigma})\right]\right. \\
& +\left[-\frac{3}{2 \kappa}\left(\Gamma_{\mu, \nu \rho}^{E}-\Gamma_{\nu, \mu \rho}^{E}\right)+\frac{4}{\kappa} B_{\mu \nu \sigma}\left(G^{-1} b\right)^{\sigma}{ }_{\rho}\right] \\
& \left.\times\left[\tilde{x}^{\rho}(\sigma)-\tilde{x}^{\rho}(\bar{\sigma})\right]\right\} \theta(\sigma-\bar{\sigma}) .
\end{aligned}
$$

After two-dimensional reparametrization, the $\sigma$ dependent part takes the form

$\left[X^{\mu}(f(\sigma))-X^{\mu}(f(\bar{\sigma}))\right] \theta[f(\sigma)-f(\bar{\sigma})]$,

where $f(\sigma)$ is a monotonically increasing function with properties $f(0)=0$ and $f(2 \pi)=2 \pi$. Therefore, the PB between different points is not reparametrization invariant. For fixed points, it can be fit to be arbitrary small, by the appropriate choice of the function $f(\sigma)$. So, only PB's at the same point are physically significant.

Taking $\sigma=\bar{\sigma}$ we find that all PB's vanish, and consequently the coordinates commute. But, taking $\sigma=\bar{\sigma}+2 \pi$ in the non-commutativity relation between the dual coordinates y's (3.11), we obtain the closed string non-commutativity relation

$\left\{y_{\mu}(\sigma+2 \pi), y_{\nu}(\sigma)\right\} \cong-\frac{2 \pi}{\kappa} B_{\mu \nu \rho} N^{\rho}$.

Here, $N^{\mu}=\frac{1}{2 \pi}\left[x^{\mu}(\sigma+2 \pi)-x^{\mu}(\sigma)\right]$ is the winding number of the original coordinates. In Sec. 4 , we will compare this relation with the result of Refs. [16,18-20].

Similarly, from Eqs. (3.12) and (3.13), we obtain

$$
\begin{array}{r}
\left\{y_{\mu}(\sigma+2 \pi), \tilde{y}_{\nu}(\sigma)\right\}+\left\{y_{\mu}(\sigma), \tilde{y}_{\nu}(\sigma+2 \pi)\right\} \cong \\
-\frac{4 \pi}{\kappa^{2}} B_{\mu \nu \rho} p^{\rho}+\frac{\pi}{\kappa}\left(3 \Gamma_{\rho, \mu \nu}^{E}-8 B_{\mu \nu \lambda} b_{\rho}^{\lambda}\right) N^{\rho},
\end{array}
$$

and

$$
\begin{aligned}
& \left\{\tilde{y}_{\mu}(\sigma+2 \pi), \tilde{y}_{\nu}(\sigma)\right\} \\
& \cong \\
& \left.\quad+3\left(\Gamma_{\mu, \nu \lambda}^{E}-\Gamma_{\nu, \mu \lambda}^{E}\right) b_{\rho}^{\lambda}\right] N^{\rho} \\
& \quad+\frac{\pi}{\kappa^{2}}\left[3\left(\Gamma_{\mu, \nu \rho}^{E}-\Gamma_{\nu, \mu \rho}^{E}\right) p^{\rho}-8 B_{\mu \nu \lambda} b_{\rho}^{\lambda}\right] p^{\rho} .
\end{aligned}
$$

Using Eq. (3.7) and integrating from $\sigma$ to $\sigma+2 \pi$ we have

$$
\begin{aligned}
& \frac{1}{2 \pi} {\left[\tilde{x}^{\mu}(\sigma+2 \pi)-\tilde{x}^{\mu}(\sigma)\right] } \\
&=\frac{1}{\kappa}\left(G^{-1}\right)^{\mu \nu} p_{v}+2\left(G^{-1}\right)^{\mu \rho} b_{\rho \lambda} N^{\lambda},
\end{aligned}
$$

where

$p_{\mu}=\frac{1}{2 \pi} \int_{\sigma}^{\sigma+2 \pi} \mathrm{d} \eta \pi_{\mu}(\eta)$.

To complete the algebra, using the expressions (2.22) and (2.23) and after one $\sigma$ integration, we find that the algebra of $y_{\mu}, \tilde{y}_{\mu}$ and ${ }^{\star} \pi^{\mu}$ is of the following form:

$$
\begin{aligned}
& \left\{y_{\mu}(\sigma),{ }^{\star} \pi^{v}(\bar{\sigma})\right\} \cong \delta_{\mu}{ }^{\nu} \delta(\sigma-\bar{\sigma})+\kappa h_{\mu \rho}[x(\sigma)] \theta_{0}^{\rho v} \times \delta \\
& (\sigma-\bar{\sigma})+\kappa h_{\mu \rho}\left[x^{\prime}(\bar{\sigma})\right] \theta_{0}^{\rho v} \theta(\sigma-\bar{\sigma}), \\
& \left\{\tilde{y}_{\mu}(\sigma),{ }^{\star} \pi^{\nu}(\bar{\sigma})\right\} \\
& \cong\left[-2 b G^{-1}-3 h[x(\sigma)] G^{-1}-2 \kappa b h[x(\sigma)] \theta_{0}\right]_{\mu}^{v} \delta(\sigma-\bar{\sigma}) \\
& -\left[3 h\left[x^{\prime}(\bar{\sigma})\right] G^{-1}+2 \kappa b h\left[x^{\prime}(\bar{\sigma})\right] \theta_{0}\right]_{\mu}^{v} \theta(\sigma-\bar{\sigma}), \\
& \left\{{ }^{\star} \pi^{\mu}(\sigma),{ }^{\star} \pi^{\nu}(\bar{\sigma})\right\} \cong 0 \text {. }
\end{aligned}
$$

Note that at the zeroth order one has $\left\{y_{\mu}(\sigma),{ }^{\star} \pi^{v}(\bar{\sigma})\right\}=$ $\delta_{\mu}^{\nu} \delta(\sigma-\bar{\sigma})$ and $\left\{\tilde{y}_{\mu}(\sigma),{ }^{\star} \pi^{\nu}(\bar{\sigma})\right\}=-2 b_{\mu}^{\nu} \delta(\sigma-\bar{\sigma})$, so both doubled space variables $y_{\mu}$ and $\tilde{y}_{\mu}$ have a nontrivial PB with ${ }^{\star} \pi^{\mu}$.

\section{Comparison with the previous results}

Let us mention that the case considered in the present paper is different from that of Ref.[16]. In Ref.[16], the 
non-commutativity relations in the nongeometric background with $Q$-flux where established, which are given in terms of winding numbers on the twisted torus $N^{3}=$ $\frac{1}{2 \pi}\left(Y^{3}(\sigma+2 \pi)-Y^{3}(\sigma)\right)$. In the present article, the noncommutativity of the nongeometric background, which is not standard even locally and for $D=3$ turns to R-flux background, was obtained in terms of the winding numbers on the torus with $H$-flux $N^{\mu}=\frac{1}{2 \pi}\left(X^{\mu}(\sigma+2 \pi)-X^{\mu}(\sigma)\right)$.

\subsection{The brief overview of the results of Ref. [16]}

Before comparing the results of our paper with those of Ref. [16] let us shortly reexpress the result of Ref. [16] using its notation. From the last identification in Eq. (2.17) and the first relation in (2.25) of Ref. [16] it follows that

$Y_{H}^{1}=Y_{0}^{2} Y_{0}^{3}+\cdots$.

Using the expression for $G_{a b}\left(Y_{3}\right)$ for the twisted torus (Table 1) of Ref. [16] we find

$\pi_{1}=\dot{Y}^{1}-H Y_{0}^{3} \dot{Y}_{0}^{2}, \quad \pi_{2}=\dot{Y}^{2}-H Y_{0}^{3} \dot{Y}_{0}^{1}$,

and consequently

$\pi_{01}=\dot{Y}_{0}^{1}, \quad \pi_{H 2}=\dot{Y}_{H}^{2}-Y_{0}^{3} \dot{Y}_{0}^{1}=\dot{Y}_{H}^{2}-Y_{0}^{3} \pi_{01}$.

The $T_{2}$-duality along $Y^{2}$, from the twisted torus to the nongeometric background produces

$$
\begin{aligned}
Z^{1} & \cong Y^{1}=Y_{0}^{1}+H Y_{0}^{2} Y_{0}^{3}, \quad Z^{2^{\prime}} \cong \dot{Y}^{2}-H Y_{0}^{3} \dot{Y}_{0}^{1} \\
& =\pi_{2}=\pi_{02}+H\left(\dot{Y}_{H}^{2}-Y_{0}^{3} \pi_{01}\right) .
\end{aligned}
$$

Thus, we find the PB

$$
\begin{aligned}
& \left\{Z^{1}(\sigma), Z^{2^{\prime}}(\bar{\sigma})\right\} \cong\left\{Y^{1}(\sigma), \pi_{2}(\bar{\sigma})\right\} \\
& \quad=H\left[Y_{0}^{3}(\sigma)-Y_{0}^{3}(\bar{\sigma})\right] \delta_{2 \pi}(\sigma-\bar{\sigma}) .
\end{aligned}
$$

Note that $\delta_{2 \pi}(\sigma-\bar{\sigma})$ is a $2 \pi$ periodic $\delta$-function, $\delta_{2 \pi}(\alpha)=$ $\sum_{n \in Z} \delta(\alpha-2 \pi n)$, so the periodic parts in the bracket in front of the $\delta$-function disappear and we obtain

$\left\{Z^{1}(\sigma), Z^{\prime 2}(\bar{\sigma})\right\}=H N^{3}(\sigma-\bar{\sigma}) \delta_{2 \pi}(\sigma-\bar{\sigma})$.

Here $N^{3}$ is the winding number of $Y_{0}^{3}$, which has the general form

$Y_{0}^{3}(\sigma)=N^{3} \sigma+Y_{\text {periodic }}^{3}(\sigma)$.

The expression $\alpha \delta_{2 \pi}(\alpha)$ is zero for $\alpha=0$, but it is different from zero for $\alpha=2 n \pi(n \in Z, n \neq 0)$.

The integration over $\bar{\sigma}$, from $\bar{\sigma}_{0}$ to $\bar{\sigma}$, produces

$$
\begin{aligned}
& \left\{Z^{1}(\sigma), Z^{2}(\bar{\sigma})\right\}-\left\{Z^{1}(\sigma), Z^{2}\left(\bar{\sigma}_{0}\right)\right\} \\
& =-\frac{1}{2 \pi} H N^{3}\left[F(\sigma-\bar{\sigma})-F\left(\sigma-\bar{\sigma}_{0}\right)\right],
\end{aligned}
$$

where

$2 \pi \int_{\alpha_{0}}^{\alpha} \mathrm{d} \eta \eta \delta_{2 \pi}(\eta)=F(\alpha)-F\left(\alpha_{0}\right)$,

and

$F(\alpha)=\sum_{n \neq 0} \frac{1}{n^{2}} e^{-i n \alpha}+i \alpha \sum_{n \neq 0} \frac{1}{n} e^{-i n \alpha}+\frac{\alpha^{2}}{2}$.

The function $F(\alpha)$ is even, $F(-\alpha)=F(\alpha)$, and $F(0)=\frac{\pi^{2}}{3}$. So, the result for the PB itself,

$\left\{Z^{1}(\sigma), Z^{2}(\bar{\sigma})\right\}=-\frac{1}{2 \pi} H N^{3}[F(\sigma-\bar{\sigma})+C]$,

is in fact equation (4.41) of Ref. [16] up to some integration constant $C$. The undetermined constant $C$ corresponds to the contribution of the zero modes of the undetermined commutators, because one started with the $\sigma$-derivative of the coordinate $Z^{2}$. The choice of Ref. [16] in subsection 4.4.2 is $C=0$, which produces the expression (4.41) of Ref. [16] and the non-commutativity at the same point, $\sigma=\bar{\sigma}$,

$\left\{Z^{1}(\sigma), Z^{2}(\sigma)\right\}=-\frac{1}{2 \pi} H N^{3} F(0)=-\frac{\pi}{6} H N^{3}$.

As was pointed out in Ref. [16], 'other reasonings could as well be pursued'. Following the line of our paper one may require that coordinates are commutative at the same point $(\sigma=\bar{\sigma})$, which produces

$C=-F(0)=-\frac{\pi^{2}}{3}$.

Thus, with this choice one has

$\left\{Z^{1}(\sigma), Z^{2}(\bar{\sigma})\right\}=H N^{3}\left[F(\sigma-\bar{\sigma})-\frac{\pi^{2}}{3}\right]$,

and one obtains the non-commutativity for $\sigma=2 \pi+\bar{\sigma}$,

$\left\{Z^{1}(\sigma+2 \pi), Z^{2}(\sigma)\right\}=\pi H N^{3}$.

\subsection{Similarities and differences}

Although we analyzed the different cases, let us compare some general features of the results considered. In both approaches the commutators are infinitesimally small and they close on some winding numbers. Note that, in general, we can connect any geometric background with every nongeometric background from the chain of T-duality (1.2). Using the T-duality transformations we can calculate the noncommutativity of the coordinates of the nongeometric background in terms of the winding numbers of the geometrical background.

For arbitrary $\sigma$ and $\bar{\sigma}$, the $\sigma$-dependence is different. In Ref. [16], up to the integration constant $C$, it is equal to

$F(\sigma-\bar{\sigma})+C$, 
and in the present article, up to the integration constant $C_{1}$, it is

$\left[x^{\mu}(\sigma)-x^{\mu}(\bar{\sigma})\right] \theta(\sigma-\bar{\sigma})+C_{1}$.

The constants appear because in both approaches we started with the sigma derivatives of the coordinates. In the papers considered, the values of the constants are taken to be $C=$ 0 and $C_{1}=0$. For these choices, the non-commutativity appears for $\sigma=\bar{\sigma}$ in Ref. [16] and for $\sigma=\bar{\sigma}+2 \pi$ in the present article. For the other choice, $C=-F(0)=-\frac{\pi^{2}}{3}$ and $C_{1}=0$, in both cases the coordinates commute at the same point $\sigma=\bar{\sigma}$ and have nontrivial $\mathrm{PB}$ for $\sigma=\bar{\sigma}+2 \pi$.

The main difference between the two approaches is the origin of non-commutativity. The nontrivial boundary conditions given in Eq. (2.25) of Ref. [16] are the source of the non-commutativity in that article. Because Ref. [16] does not consider $T_{3}$-dualization, the $\beta_{\mu}^{0}$-functions (introduced in Eq. (2.15)) are zero and there is no non-commutativity of this kind. On the other hand, in the case considered in this paper, just these $\beta_{\mu}^{0}$ functions are the sources of the noncommutativity, even in the absence of the nontrivial boundary conditions of Ref. [16]. For complete non-commutativity relations one should take into account both kinds of noncommutativity.

\section{Concluding remarks}

In the present article we derived the closed string noncommutativity relations. We considered the theory describing a string moving in a weakly curved background. Its Tdual theory is obtained performing the T-dualization procedure along all the coordinates [23]. The T-dual transformation laws play a central role in our approach. These laws connect the world-sheet derivatives of the coordinates and momenta in the original and the T-dual theory. The zero orders are transformation laws of the constant background and they do not lead to the non-commutativity. The term $\beta_{\mu}^{0}$, which is infinitesimally small and bilinear in the $x^{\mu}$ coordinates, plays a key role in obtaining the non-commutativity relations.

In the original space we choose the standard Poisoon brackets. The T-dual coordinates $y_{\mu}$ have two terms: one linear in the original momenta and the other bilinear in the original coordinates. This explains the nontrivial $\mathrm{PB}\left\{y_{\mu}, y_{\nu}\right\}$ of Eq. (3.11), which is linear in the coordinates. Note that in the case of an open string moving in the flat background coordinate is linear function in both effective momenta and coordinates. Therefore, the corresponding PB is constant.

The T-dual momenta ${ }^{\star} \pi^{\mu}$ are bilinear expressions in the original coordinates. Thus, the PB of the T-dual momenta vanishes, see Eq. (3.21), but the PB between the T-dual coor- dinates and the momenta (3.19) obtained an additional term linear in the coordinates.

In the doubled space there exists the additional coordinate $\tilde{y}_{\mu}$. It consists of a term linear in the original momenta, but with the coefficient linear in the original coordinate and the other terms bilinear in the original coordinates. Thus, it produces a nontrivial PB with all variables $\left(y_{\mu}, \tilde{y}_{\mu},{ }^{\star} \pi^{\mu}\right)$, see Eqs. (3.12), (3.13), and (3.20).

The general structure of the non-commutativity relations is

$$
\begin{aligned}
& \left\{Y_{\mu}(\sigma), Y_{v}(\bar{\sigma})\right\}=\left\{F_{\mu \nu \rho}\left[x^{\rho}(\sigma)-x^{\rho}(\bar{\sigma})\right]\right. \\
& \left.+\tilde{F}_{\mu \nu \rho}\left[\tilde{x}^{\rho}(\sigma)-\tilde{x}^{\rho}(\bar{\sigma})\right]\right\} \theta(\sigma-\bar{\sigma}),
\end{aligned}
$$

where $Y_{\mu}=\left(y_{\mu}, \tilde{y}_{\nu}\right)$ and $F_{\mu \nu \rho}$ and $\tilde{F}_{\mu \nu \rho}$ are the constant and infinitesimally small fluxes. At the same points, for $\sigma=\bar{\sigma}$ all PB's are zero. In the important particular case for $\sigma=\bar{\sigma}+2 \pi$ we get

$$
\begin{aligned}
& \left\{Y_{\mu}(\sigma+2 \pi), Y_{\nu}(\sigma)\right\} \\
& \quad=2 \pi\left[\left(F_{\mu \nu \rho}+2 \tilde{F}_{\mu \nu \alpha} b_{\rho}^{\alpha}\right) N^{\rho}+\frac{1}{\kappa} \tilde{F}_{\mu \nu}{ }^{\rho} p_{\rho}\right],
\end{aligned}
$$

where $N^{\mu}$ and $p_{\mu}$ are the winding numbers and momenta of the original theory. We rewrite it in the form

$$
\begin{aligned}
& \left\{Y_{\mu}(\sigma+2 \pi), Y_{\nu}(\sigma)\right\} \\
& \quad=\oint_{C_{\rho}} F_{\mu \nu \rho} \mathrm{d} x^{\rho}+\oint_{\tilde{C}_{\rho}} \tilde{F}_{\mu \nu \rho} \mathrm{d} \tilde{x}^{\rho},
\end{aligned}
$$

where $C_{\rho}$ and $\tilde{C}_{\rho}$ are cycles around which the closed string is wrapped. Note that the 'wrapping' of the auxiliary coordinate $\tilde{x}^{\mu}$ is in accordance with Eq. (3.17) and represents a linear combination of momenta $p_{\mu}$ and winding numbers $N^{\mu}$. This generalizes the conjecture of Ref. [32] on the relation between the closed string non-commutativity and fluxes.

In terms of Ref. [16] for the three-dimensional torus $x^{\mu} \rightarrow X^{a},(a=1,2,3)$ our case corresponds to the noncommutativity of the nongeometric background with $W^{a}$ coordinates and $R$-fluxes obtained after the successive performation of all three T-dualizations along all three coordinates. It relates the $W^{a}$ with the $X^{a}$ coordinates of the torus with $H$-flux, and so the PB closes on the winding number of the $X^{a}$-coordinates. We hope that these results will contribute to a better understanding of the strangest, uncommon R-flux configurations where the non-commutativity appears as a consequence of the nontrivial $\beta_{\mu}^{0}$-functions. Note that Ref. [16] uses $T_{2}$-duality (performed along $Y^{2}$ ) and the relation $Z^{a}=Z^{a}\left(Y^{a}\right)$ to obtain the non-commutativity of the nongeometric background with $Q$-flux in terms of the winding of the $Y^{a}$-coordinates. There the non-commutativity originates from the nontrivial boundary conditions. To obtain the general structure of the closed string non-commutativity for arbitrary background of the chain (1.2) one should find its 
T-duality transformations with all other backgrounds of the chain and calculate both kinds of non-commutativity originating from nontrivial boundary conditions as well as from nontrivial $\beta_{\mu}^{0}$ functions.

The term of the action with the constant part of the KalbRamond field $b_{\mu \nu}$ is topological. Thus, it does not contribute to the equations of motion. In the open string case it contributes to the boundary conditions and it is a source of the open string non-commutativity. In the closed string case it is absent from boundary conditions as well. Classically, we can gauge it away and the Kalb-Ramond field becomes infinitesimally small. But if $b_{\mu \nu}=0$ one loses topological contributions. In order to investigate the global structure of the theory with holonomies of the world-sheet gauge fields in quantum theory we should preserve such a term.

Putting $b_{\mu \nu}=0$ the non-commutativity relations (3.14), (3.15), and (3.16) get a simpler form,

$$
\begin{aligned}
& \left\{y_{\mu}(\sigma+2 \pi), y_{\nu}(\sigma)\right\}=-\frac{2 \pi}{\kappa} B_{\mu \nu \rho} N^{\rho}, \\
& \left\{y_{\mu}(\sigma+2 \pi), \tilde{y}_{\nu}(\sigma)\right\}=-\frac{1}{\kappa} G_{\mu \nu}-\frac{2 \pi}{\kappa^{2}} B_{\mu \nu}{ }^{\rho} p_{\rho}, \\
& \left\{\tilde{y}_{\mu}(\sigma+2 \pi), \tilde{y}_{\nu}(\sigma)\right\}=-\frac{6 \pi}{\kappa} B_{\mu \nu \rho} N^{\rho} .
\end{aligned}
$$

Acknowledgments Work supported in part by the Serbian Ministry of Education, Science and Technological Development, under contract No. 171031

Open Access This article is distributed under the terms of the Creative Commons Attribution License which permits any use, distribution, and reproduction in any medium, provided the original author(s) and the source are credited.

Funded by $\mathrm{SCOAP}^{3}$ / License Version CC BY 4.0.

\section{Appendix A: The momentum in T-dual theory}

Let us here calculate the T-dual momentum given in Eq. (2.18). The T-dual theory depends on the two variables $y_{\mu}, \tilde{y}_{\mu}$, which are connected by the relations $\dot{y}_{\mu}=\tilde{y}_{\mu}^{\prime}$, $y_{\mu}^{\prime}=\dot{\tilde{y}}_{\mu}$. Therefore, to obtain the momentum canonically conjugated to $y_{\mu}$, we should vary the action with respect to both $\dot{y}_{\mu}$ and $\tilde{y}_{\mu}^{\prime}$.

First, let us calculate the contribution from the background fields argument. With the help of the relation

$\Theta_{-}^{\mu v}[x]=\Theta_{0-}^{\mu \nu}-2 \kappa \Theta_{0-}^{\mu \rho} h_{\rho \sigma}[x] \Theta_{0-}^{\sigma v}$,

we rewrite the T-dual action (2.8) as

$$
\begin{aligned}
{ }^{\star} S[y] & ={ }^{\star} S_{0}-\kappa^{3} \int \mathrm{d}^{2} \xi \partial_{+} y_{\mu} \Theta_{0-}^{\mu \rho} h_{\rho \sigma}[\Delta V[y]] \Theta_{0-}^{\sigma \nu} \partial_{-} y_{\nu}, \\
{ }^{\star} S_{0} & =\frac{\kappa^{2}}{2} \int \mathrm{d}^{2} \xi \partial_{+} y_{\mu} \Theta_{0-}^{\mu \nu} \partial_{-} y_{\nu} .
\end{aligned}
$$

Using the expression

$\partial_{ \pm} V^{\mu}=-\kappa \Theta_{0 \pm}^{\mu \nu} \partial_{ \pm} y_{v}^{(0)}$

we obtain

$$
\begin{aligned}
{ }^{\star} S[y] & ={ }^{\star} S_{0}+\kappa \int \mathrm{d}^{2} \xi \partial_{+} V^{\mu} h_{\mu \nu}[\Delta V] \partial_{-} V^{\nu} \\
& ={ }^{\star} S_{0}+\kappa \int \mathrm{d}^{2} \xi \Delta V^{\mu} h_{\mu \nu}\left[\partial_{-} V\right] \partial_{+} V^{\nu} .
\end{aligned}
$$

Because of the relation

$h_{\mu \nu}\left[\partial_{-} V\right] \partial_{+} V^{\nu}=\partial_{0} \beta_{\mu}^{0}[V]+\partial_{1} \beta_{\mu}^{1}[V]$,

the action becomes

$$
\begin{aligned}
{ }^{\star} S[y]= & { }^{\star} S_{0}+\kappa \int \mathrm{d}^{2} \xi\left[\kappa \Delta y_{\mu} \theta_{0}^{\mu \nu}+\Delta \tilde{y}_{\mu}\left(g^{-1}\right)^{\mu \nu}\right] \\
& \times\left(\partial_{0} \beta_{v}^{0}[V]+\partial_{1} \beta_{\nu}^{1}[V]\right) .
\end{aligned}
$$

So, the contribution to the T-dual momentum coming from the T-dual background fields argument is obtained from Eq. (6.6), integrating over $\sigma$ by parts in $\Delta \tilde{y}_{\mu}\left(g^{-1}\right)^{\mu v} \partial_{1} \beta_{v}^{1}$. Using $\tilde{y}_{\mu}^{\prime}=\dot{y}_{\mu}$ we obtain

$\Delta^{\star} \pi^{\mu}=-\kappa\left(g^{-1}\right)^{\mu \nu} \beta_{v}^{1}[V]$.

Therefore, the total T-dual momentum is

$$
\begin{aligned}
{ }^{\star} \pi^{\mu}= & \kappa\left(G_{E}^{-1}\right)^{\mu v}[\Delta V[y]] \dot{y}_{v} \\
& -\kappa^{2} \theta^{\mu \nu}[\Delta V[y]] y_{v}^{\prime}-\kappa\left(g^{-1}\right)^{\mu v} \beta_{v}^{1}[V[y]] .
\end{aligned}
$$

\section{Appendix B: Fluxes}

The field strength of the original Kalb-Ramond field is given by Eq. (2.5). The original metric $G_{\mu \nu}$ is constant, and therefore the corresponding Christoffel connection is zero. The effective metric $G_{\mu \nu}^{E}$ is linear in the coordinates and the corresponding Christoffel connection,

$$
\begin{aligned}
\Gamma_{\mu, \nu \rho}^{E} & =\frac{1}{2}\left(\partial_{\nu} G_{\mu \rho}^{E}+\partial_{\rho} G_{\mu \nu}^{E}-\partial_{\mu} G_{\nu \rho}^{E}\right) \\
& =-\frac{4}{3}\left(B_{\mu \sigma \nu}\left(G^{-1} b\right)_{\rho}^{\sigma}+B_{\mu \sigma \rho}\left(G^{-1} b\right)_{\nu}^{\sigma}\right),
\end{aligned}
$$

is an infinitesimally small constant. It will be used in the following form:

$$
\Gamma_{\mu, v \rho}^{E} x^{\mu}=4\left(h[x] G^{-1} b+b G^{-1} h[x]\right)_{\nu \rho}
$$

and

$$
\begin{aligned}
& \left(\Gamma_{\mu, \nu \rho}^{E}-\Gamma_{\nu, \mu \rho}^{E}\right) x^{\rho}=8 h_{\mu \nu}[b x] \\
& \quad-4\left(h[x] G^{-1} b-b G^{-1} h[x]\right)_{\mu \nu} .
\end{aligned}
$$

We express the dual Kalb-Ramond field [23] as

${ }^{\star} B^{\mu \nu}[\Delta V]={ }^{\star} b^{\mu \nu}+Q_{\rho}^{\mu \nu} \Delta V^{\rho}$, 
where ${ }^{\star} b^{\mu \nu}=\frac{\kappa}{2} \theta_{0}^{\mu \nu}$ and

$Q_{\rho}^{\mu \nu}=-\frac{1}{3}\left[\left(g^{-1}\right)^{\mu \sigma}\left(g^{-1}\right)^{\nu \tau}-\kappa^{2} \theta_{0}^{\mu \sigma} \theta_{0}^{\nu \tau}\right] B_{\sigma \tau \rho}$.

This will be used as

$$
\begin{aligned}
Q_{\rho}^{\mu \nu} x^{\rho} & =-\left(g^{-1}\right)^{\mu \rho}\left[h[x]+4 b G^{-1} h[x] G^{-1} b\right]_{\rho \sigma}\left(g^{-1}\right)^{\sigma v} \\
& =-\left[g^{-1} h[x] g^{-1}+\kappa^{2} \theta_{0} h[x] \theta_{0}\right]^{\mu \nu}
\end{aligned}
$$

\section{Appendix C: PB's between pure coordinates}

Starting with the PB of the $\sigma$ derivatives of the coordinates

$$
\left\{X_{\mu}^{\prime}(\sigma), Y_{\nu}^{\prime}(\bar{\sigma})\right\} \cong K_{\mu \nu}^{\prime}(\sigma) \delta(\sigma-\bar{\sigma})+L_{\mu \nu}(\sigma) \delta^{\prime}(\sigma-\bar{\sigma}),
$$

let us find the expression for the PB between the coordinates, $\left\{X_{\mu}(\sigma), Y_{v}(\bar{\sigma})\right\}$. From Eq. (8.1) it follows that $\Delta X_{\mu}\left(\sigma, \sigma_{0}\right)$ and $\Delta Y_{\mu}\left(\sigma, \sigma_{0}\right)$ defined by

$$
\begin{aligned}
& \Delta X_{\mu}\left(\sigma, \sigma_{0}\right)=\int_{\sigma_{0}}^{\sigma} \mathrm{d} \eta X_{\mu}^{\prime}(\eta)=X_{\mu}(\sigma)-X_{\mu}\left(\sigma_{0}\right), \\
& \Delta Y_{\mu}\left(\sigma, \sigma_{0}\right)=\int_{\sigma_{0}}^{\sigma} \mathrm{d} \eta Y_{\mu}^{\prime}(\eta)=Y_{\mu}(\sigma)-Y_{\mu}\left(\sigma_{0}\right)
\end{aligned}
$$

satisfy

$$
\begin{aligned}
& \left\{\Delta X_{\mu}\left(\sigma, \sigma_{0}\right), \Delta Y_{\nu}\left(\bar{\sigma}, \bar{\sigma}_{0}\right)\right\} \\
& \quad \cong \int_{\sigma_{0}}^{\sigma} \mathrm{d} \eta \int_{\bar{\sigma}_{0}}^{\bar{\sigma}} d \bar{\eta}\left[K_{\mu \nu}^{\prime}(\eta) \delta(\eta-\bar{\eta})+L_{\mu \nu}(\eta) \delta^{\prime}(\eta-\bar{\eta})\right] .
\end{aligned}
$$

Integrating over $\bar{\eta}$ and using

$$
\begin{aligned}
& \int_{\sigma_{0}}^{\sigma} \mathrm{d} \eta f(\eta) \delta(\eta-\bar{\sigma}) \\
& \quad=f(\bar{\sigma})\left[\theta(\sigma-\bar{\sigma})-\theta\left(\sigma_{0}-\bar{\sigma}\right)\right],
\end{aligned}
$$

we obtain

$$
\begin{aligned}
& \left\{\Delta X_{\mu}\left(\sigma, \sigma_{0}\right), \Delta Y_{\nu}\left(\bar{\sigma}, \bar{\sigma}_{0}\right)\right\} \\
& \cong \int_{\sigma_{0}}^{\sigma} \mathrm{d} \eta\left[K_{\mu \nu}^{\prime}(\eta)\left[\theta\left(\eta-\bar{\sigma}_{0}\right)-\theta(\eta-\bar{\sigma})\right]\right. \\
& \left.\quad+L_{\mu \nu}(\eta)\left[\delta\left(\eta-\bar{\sigma}_{0}\right)-\delta(\eta-\bar{\sigma})\right]\right],
\end{aligned}
$$

where the function $\theta(\sigma)$ is defined as

$$
\theta(\sigma) \equiv \int_{0}^{\sigma} \mathrm{d} \eta \delta(\eta)=\frac{1}{2 \pi}\left(\sigma+2 \sum_{n \geq 1} \frac{1}{n} \sin n \sigma\right)
$$

$$
= \begin{cases}0 & \text { if } \sigma=0 \\ 1 / 2 & \text { if } 0<\sigma<2 \pi, \quad \sigma \in[0.2 \pi] . \\ 1 & \text { if } \sigma=2 \pi\end{cases}
$$

Integrating by parts over $\eta$ and using Eq. (8.4) we get

$$
\begin{aligned}
&\left\{\Delta X_{\mu}\left(\sigma, \sigma_{0}\right), \Delta Y_{\nu}\left(\bar{\sigma}, \bar{\sigma}_{0}\right)\right\} \\
& \cong K_{\mu \nu}(\sigma)\left[\theta\left(\sigma-\bar{\sigma}_{0}\right)-\theta(\sigma-\bar{\sigma})\right] \\
&-K_{\mu \nu}\left(\sigma_{0}\right)\left[\theta\left(\sigma_{0}-\bar{\sigma}_{0}\right)-\theta\left(\sigma_{0}-\bar{\sigma}\right)\right] \\
&-K_{\mu \nu}\left(\bar{\sigma}_{0}\right)\left[\theta\left(\sigma-\bar{\sigma}_{0}\right)-\theta\left(\sigma_{0}-\bar{\sigma}_{0}\right)\right] \\
&+K_{\mu \nu}(\bar{\sigma})\left[\theta(\sigma-\bar{\sigma})-\theta\left(\sigma_{0}-\bar{\sigma}\right)\right] \\
&+L_{\mu \nu}\left(\bar{\sigma}_{0}\right)\left[\theta\left(\sigma-\bar{\sigma}_{0}\right)-\theta\left(\sigma_{0}-\bar{\sigma}_{0}\right)\right] \\
&-L_{\mu \nu}(\bar{\sigma})\left[\theta(\sigma-\bar{\sigma})-\theta\left(\sigma_{0}-\bar{\sigma}\right)\right] .
\end{aligned}
$$

The relation

$$
\begin{aligned}
& \left\{X_{\mu}(\tau, \sigma), Y_{\nu}(\tau, \bar{\sigma})\right\} \cong-\left[K_{\mu \nu}(\sigma)-K_{\mu \nu}(\bar{\sigma})+L_{\mu \nu}(\bar{\sigma})\right] \\
& \quad \times \theta(\sigma-\bar{\sigma})
\end{aligned}
$$

solves Eq. (8.7), up to additive constant.

For $X_{\mu}=Y_{\mu}$, the antisymmetry of the left hand side under the replacement $\mu \leftrightarrow \nu$ and $\sigma \leftrightarrow \bar{\sigma}$ produces the conditions $L_{\mu \nu}=L_{v \mu}$ and $K_{\mu \nu}+K_{\nu \mu}=L_{\mu \nu}$.

\section{References}

1. F. Ardalan, H. Arfaei, M.M. Sheikh-Jabbari, JHEP 02, 016 (1999)

2. C.S. Chu, P.M. Ho, Nucl. Phys. B 550, 151 (1999)

3. N. Seiberg, E. Witten, JHEP 09, 032 (1999)

4. F. Ardalan, H. Arfaei, M.M. Sheikh-Jabbari, Nucl. Phys. B 576, 578 (2000)

5. C.S. Chu, P.M. Ho, Nucl. Phys. B 568, 447 (2000)

6. T. Lee, Phys. Rev. D 62, 024022 (2000)

7. B. Sazdović, Eur. Phys. J. C 44, 599 (2005)

8. B. Nikolić, B. Sazdović, Phys. Rev. D 74, 045024 (2006)

9. B. Nikolić, B. Sazdović, Phys. Rev. D 75, 085011 (2007)

10. B. Nikolić, B. Sazdović, Adv. Theor. Math. Phys. 14, 1 (2010)

11. Lj. Davidović, B. Sazdović, Phys. Rev. D 83, 066014 (2011)

12. Lj. Davidović, B. Sazdović, JHEP 08, 112 (2011)

13. T.H. Buscher, Phys. Lett. B 194, 59 (1987)

14. T.H. Buscher, Phys. Lett. B 201, 466 (1988)

15. D. Luest, JHEP 12, 084 (2010)

16. D. Andriot, M. Larfors, D. Luest, P. Patalong, JHEP 06, 021 (2013)

17. D. Andriot, O. Hohm, M. Larfors, D. Luest, P. Patalong, Phys. Rev. Lett. 108, 261602 (2012)

18. D. Luest, arxiv:1205.0100 [hep-th]

19. R. Blumenhagen, A. Deser, D. Luest, E. Plauschinn, F. Rennecke, J. Phys. A 44, 385401 (2011)

20. C. Condeescu, I. Florakis, D. Luest, JHEP 04, 121 (2012)

21. J. Shelton, W. Taylor, B. Wecht, JHEP 10, 085 (2005)

22. A. Dabholkar, C. Hull, JHEP 05, 009 (2006)

23. Lj. Davidović, B. Sazdović, Eur. Phys. J. C 74 (1), (2014) 
24. R. Blumenhagen, E. Plauschinn, J. Phys. A Math. Theor. 44, 015401 (2011)

25. E.S. Fradkin, A.A. Tseytlin, Phys. Lett. B 158, 316 (1985)

26. E.S. Fradkin, A.A. Tseytlin, Nucl. Phys. B 261, 1 (1985)

27. K. Becker, M. Becker, J. Schwarz, String Theory and M-Theory: A Modern Introduction. (Cambridge University Press, Cambridge, 2006)

28. B. Zwiebach, A First Course in String Theory. (Cambridge University Press, Cambridge, 2004)
29. V. Schomerus, Class. Quant. Grav. 19, 5781 (2002)

30. L. Cornalba, R. Schiappa, Commun. Math. Phys. 225, 33 (2002)

31. Lj. Davidović, B. Sazdović, EPJ C 72 (11), 2199 (2012)

32. D. Andriot, O. Hohm, M. Larfors, D. Luest,, P. Patalong, Fort. Phys. 60 (2012) 